\title{
Research on convexity-preserving of the weight factor of cubic NURBS interpolation curve
}

\author{
LIU Jiyuan ${ }^{1, a}$, CHEN Shaoping ${ }^{\star 2, b}$ \\ ${ }^{1}$ College of science, Wuhan University Of Technology, 430070Wuhan,China \\ ${ }^{2}$ College of science, Wuhan University Of Technology, 430070Wuhan,China \\ a676206196@qq.com, b chensp@whut.edu.cn
}

Keywords: NURBS interpolation curve; weight factor; convexity- preserving

\begin{abstract}
This paper mainly discusses the weight factor that influence on cubic NURBS interpolation curve shape. Based on the existing curve and surface interpolation, the thought, the criterion and the concrete steps of the convexity-preserving of the weight factor of cubic NURBS interpolation curve are provided, numerical examples are carried out by the computer simulation and the weights are controlled with purpose and procedure. Then a scope of the convexitypreserving is putted up with through observing the changes of the cubic NURBS interpolation curve and the associated with this operation. In addition, its application in the design of ship modeling is given.
\end{abstract}

\section{Introduction}

NURBS curve and surface are used widely, it is very important to illustrate how to modify the existing curve and surface to meet the requirements of the designer after constructing the NURBS curve[1]. The NURBS curve is defined by the control points, the weight factor, and the node vector. Using the partial modification of the NURBS curve, the weight factor and its corresponding control vertices only affect the shape of the curve in the in $u \in\left[u_{i}, u_{i+p+1}\right]$, it makes the NURBS method more flexible. We can get better effect on the convexity of the NURBS curve and control the shape of curve that is constructed by the known control of the vertices. However, it is not easy to control the shape of the curve obtained by NURBS interpolation, and to control and guarantee the convexity-preserving of the curve. There are many articles about adjusting the shape of the NURBS curve from the positive adjustment, for example: Juhsz[2]discussed the NURBS curve adjustment algorithm based on weight factor; Piegl[3,4]studied the changes when the weight factors change, and put forward the two methods that based on the modification of control points and weight factors; $\mathrm{Au}$ and Yuen[5] studied the change of the shape of the curve when the control points and weight factors are changed. However, there are few articles about adjusting the shape of the NURBS curve from the backward adjustment. Most of them are about B spline interpolation curve[6], and hardly any articles about the NURBS interpolation curves. The improper value of the weight factor may cause very bad parameters, and even destroy the shape of the curve. It is impossible to make reasonable control of the shape, and can not guarantee the convexity-preserving of NURBS interpolation curve. Until now, people are not completely free to use this tool. Therefore, it is an important topic to study the effect of the change of the weight factor on the convexity-preserving of NURBS interpolation curve.

Taking cubic NURBS interpolation curve as an example, this paper studies the convexity-preserving of NURBS interpolation curve. The paper puts forward a scope of the convexity-preserving of the weight factor of the cubic three NURBS curves under the guidance of the end point vector. The good convexity and smoothness of the curve can be ensured in the range. 


\section{Cubic NURBS curve}

\subsection{Cubic NURBS curve[7]}

A curve of the three NURBS is defined as

$$
C(u)=\frac{\sum_{i=0}^{n} B_{i, 3}(u) W_{i} V_{i}}{\sum_{i=0}^{n} B_{i, 3}(u) W_{i}}, 0 \leq u \leq 1
$$

Where the $V_{i}$ are control points, the $W_{i}$ are weight factor. In order to make the denominator is not zero, where specified, $w_{0}>0, w_{n}>0, w_{i} \geq 0,\left\{B_{i, 3}(u)\right\}$ is the basis function which are defined in the non periodic and non uniform node vector

$$
U=\left\{u_{0}=\cdots=u_{3}, u_{4}, \cdots u_{n}, u_{n+1}=\cdots=u_{n+1}\right\}
$$

Its recursion formula is

$$
\left\{\begin{array}{l}
B_{i, 0}(u)=\left\{\begin{array}{l}
1, u_{i} \leq u \leq u_{i+1} \\
0, \text { others }
\end{array}\right. \\
B_{i, k}(u)=\frac{u-u_{i}}{u_{i+k}-u_{i}} B_{i, k-1}(u)+\frac{u_{i+k+1}-u}{u_{i+k+1}-u_{i+1}} B_{i+1, k-1}(u) \\
\text { stipulate } \frac{0}{0}=0
\end{array}\right.
$$

\subsection{Determination of node vector of interpolation curve}

After determining the parameters $\tilde{t}_{k}$, it is necessary to determine the appropriate node vector. $U=\left\{u_{0}, u_{1}, \cdots, u_{n+p+1}\right\}$. The selection of node vector group $U$ needs to meet three conditions: (1) the distribution of the value of the type can be accurately reflected; (2) the numerical stability of the equations can be maintained; (3) the additional pilot limit can be accommodated[8]. Combined with the above conditions, this paper selects the algebraic mean value method to determine the node vector, also known as the de Boor node[9]. That is

$$
u_{i}=\left\{\begin{array}{l}
0, i=0,1, \cdots, p \\
\frac{1}{p} \sum_{j=i-p}^{i-1} \tilde{t}_{j}, i=p+1, p+2, \cdots, n \\
1, i=n+1, n+2, \cdots, n+p+1
\end{array}\right.
$$

So we construct a new node vector[10] group with constraints based on the de Boor node. The method is first to calculate the node vector group, that is the Boor de node vector group, and then add an additional node to the first and the last one to the non zero node, so as to get the node vector of the end point of the vector, the algorithm is as follows.

- Algorithm A-2.1

Input:

$$
\begin{array}{ll}
\tilde{t}_{k}, k=0,1,2, \cdots, n & / * \text { parameter value* / } \\
p & / * \text { Number of interpolation curves * / }
\end{array}
$$

Output:

$u_{i}, i=0,1, \cdots, n+p+3 \quad / *$ node vector* /

Algorithm:

$$
\begin{aligned}
& \begin{array}{l}
m=n+2 \\
\text { for } i=0 \text { to } p \text { do } \\
\quad u_{i}=\tilde{t}_{0}
\end{array} \\
& u_{m+i+1}=\tilde{t}_{n} \\
& \text { end } \\
& \text { is }=0, i e=n-p+1, r=p \\
& \text { for } i=i \text { is to ie do }
\end{aligned}
$$




$$
\begin{aligned}
& j s=\max (0, i) \\
& j e=\min (n, i+p-1) \\
& r=r+1 \\
& \text { sum }=0 \\
& \text { for } j=j s \text { to je do } \\
& \text { sum }=\text { sum }+\tilde{t}_{j} \\
& \qquad u_{r}=\frac{\text { sum }}{j e-j s+1}
\end{aligned}
$$

end

end

\section{Cubic NURBS interpolation curve convexity preserving}

\subsection{Geometric sense of the weight factor[7]}

Fixed NURBS curve of the control polygon, we can pass the weight factor to control the shape of the curve so that the NURBS curve is more flexible. However, the following question is how to adjust the power factor from the geometry. Piegl reveals the geometric meaning of the weight factor: weight is equal to the control point of a straight line are respectively provided with power factor value $\omega_{i}=+\infty, 0,1$ and the cross ratio of the four points $\omega_{i} \neq 0,1$, that is, assume the $\bar{u} \in\left[u_{i}, u_{i+p+1}\right]$ defines a point $R=C\left(\bar{u} ; w_{k}=0\right) ; \quad M=C\left(\bar{u} ; w_{k}=1\right) ; \quad p=C\left(\bar{u} ; w_{k} \neq 0,1\right), \quad w_{k}=\frac{P_{k} M}{R M} / \frac{P_{k} p}{R p}, \omega_{k}$ for this four points $P_{k}, R, M, P$ of cross ratio. As shown in figure 1

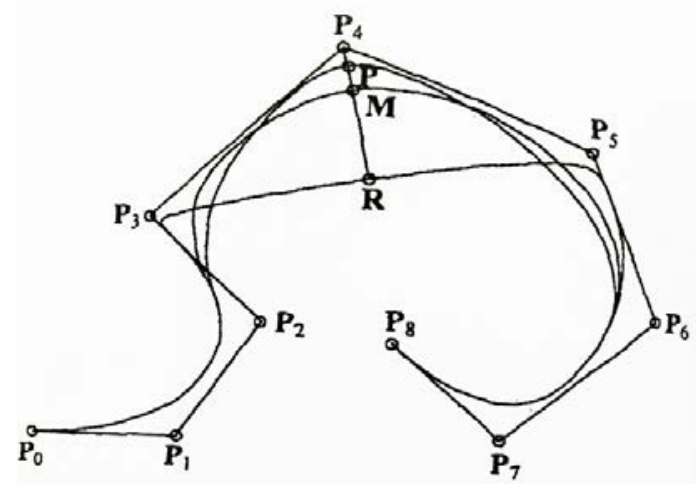

Figure 1. Geometric meaning

According to the geometric meaning of weight factor, it is easy to get the influence on shape of NURBS curve:

(1) If we fix all control points and all other weight factors except the $\omega_{i}$ are unchanged, the point $p$ is moved when the factor $\omega_{i}$ are changed. It scans a straight line through the control points in the space. When $\omega_{i}=+\infty$, the $p$ and the control point $P_{i}$ coincide.

(2) If $\omega_{i}$ is increased, the curve is drawn to the control point $P_{i}$; if $\omega_{i}$ decreases, the curve is pushed out of control point $P_{i}$. That is, the decrease or increase of weight factor leads to push and pull of the curve relative to the apex.

(3) If $\omega_{i}=0, \omega_{j} \neq 0(j=0,1,2 \cdots, i-1, i+1, \cdots, n)$ the curve on the convex hull of the $p_{0} \cdots p_{i-1} p_{i+1} \cdots p_{n}$.If $\omega_{i} \neq 0, \omega_{1}=\cdots=\omega_{j-1}=0, \cdots, \omega_{k} \neq 0, \quad \omega_{k+1}=\cdots=\omega_{n-1}=0, \omega_{j} \neq 0,(1 \leq j \leq k \leq n-1)$, then the curve on the convex hull of the $p_{0} \cdots p_{i-1} p_{i+1} \cdots p_{n}$.

If we change the weight factor $\omega_{k}$, the $P=C(\bar{u})$ can be pulled or pushed to a new location $\tilde{P}$, denoted by $d=P \tilde{P}=|P-\tilde{P}|$, Then the new weight factor can be obtained by the following formula:

$$
\tilde{w}_{k}=w_{k}\left(1 \pm \frac{d}{R_{k, p}(u) \cdot\left(P_{k} p \mp d\right)}\right)
$$


Adjusting the shape of the local curve of the point by modifying the value of the weight factor in order to the curve has a good convexity preserving, and the concrete steps are as follows:

Step1: take a point $P$ on the curve, and then take a point $Q$ of the control polygon, the system counts $\bar{u}$ to make $P=C(\bar{u})$.

Step2: if the point $Q$ is not the original control point, we use the reverse node insertion method to make it a control point, denoted by $P_{k}$. In the direction of the line $P P_{k}$ taking a point $\tilde{P}$, calculating $d$.

Step3: according to formula (5) counting the $\tilde{\omega}_{k}$.

Here is an example of a three NURBS curve to further illustration, the given control vertices, respectively $\quad P_{0}=(-0.445,0.118) P_{1}=(0.013,0.128) \quad P_{2}=(1.140,1.200) \quad P_{3}=(0.011,1.420) \quad P_{5}=(2.574,1.540)$ $P_{6}=(3.050,1.282) P_{7}=(2.013,0.131) \quad P_{8}=(1.970,0.760) \quad P_{9}=(1.920,1.193)$ the corresponding weight factors are $w_{0}=1, w_{1}=0.8, w_{2}=1, w_{3}=2.9, w_{4}=3.2, w_{5}=1,8, w_{6}=0.5, w_{7}=1.7, w_{8}=2.6, w_{9}=0.4$. According to the known data, we get NURBS curve diagram just as the solid line shows, the shape of the curve corresponding to the control point $P_{4}$ is not ideal. Below we adjust the shape of the curve by modifying the value of the corresponding weight factor $w_{4}$ to make the point $P$ on the curve move to with a right place. In accordance with the above method, the parameters are calculated $\bar{u}=0.52$, and the moving distance is $d=0.075$, the value of the new weight factor is $\tilde{\omega}_{k}=2.57$. The modified figure is shown in the dotted line, by modifying the shape of the front and rear curves, the change of the weight factor leading to effect of the curve is clearly seen.

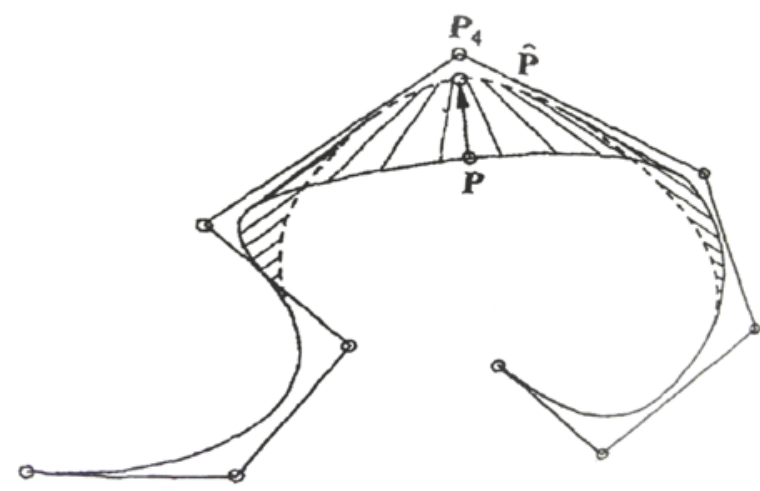

Figure 2 the changes of the shape of the three NURBS curve before and after modification

In practical application, the modified weight factor can make a small change in the shape of the curve. That is, it plays a role in fine-tuning. In figure 2, point $P$ can be pulled to control the vertex $P_{k}$, and in this operation, in which the $P_{k}$ can be inherent to limit the length of the $P$ as long as possible. In particular, when $P_{k}$ is not the original control point, we can use the inverse node insertion method to get a new control point. In addition, even if we moderate or push these vertices, we need to make a substantial change to the corresponding weight factor. However, when the weight factor is modified by a large margin, it is possible to cause very bad parameters. Therefore, when the software is implemented in this step, we need to limit the weight factor, that is, it is very important to find out the scope of the convexity-preserving of the weight factor in curve modeling technology

\subsection{The scope of the convexity-preserving of the weight factor under the constraint of the endpoint vector}

In the process of constructing curve structure, the basic method is based on the value of the known data point to operate interpolation. The existing NURBS curve interpolation method is based on the interpolation of the endpoint vector to be the boundary conditions. Although the obtained interpolation curve can achieve the desired effect, as to the high requirements of industrial design, it is difficult to meet the actual requirements, especially to the convexity-preserving. The NURBS curve obtained by interpolation method cannot guarantee its good convexity[11], so we have to make further shape adjustment of NURBS interpolation curve. The weight factor has a local effect on the shape of the curve, because the control points which corresponding to the weight factor only 
affect the shape of the curve in the interval $u \in\left[u_{i}, u_{i+p+1}\right]$, the other part of the NURBS curve is not affected, so only the part of the curve is considered.

According to the method of existing interpolation, the weight factor, the convexity-preserving theory and the constraint of curve in the design of actual production, we step to control the weight factor in the computer experiment. Then we study the imagination with this operation (that is, the change of the curve) through the observation. Then the scope of the convexity-preserving of the weight factor is discussed in order to optimize the design of curves. Specific steps are as follows:

Step1: using the chord length method to make the data points $Q_{k}$ parameterized .

Step2: determination of the node vectors of interpolation curve under the constraint of the endpoint vector.

Step3: the control points are obtained by solving the linear equations[9], then getting cubic NURBS interpolation curves through the data points $Q_{k}$ using the de Boor algorithm.

Step4: take a point $P$ on the curve, and then take a point $Q$ of the control polygon, the system counts $\bar{u}$ to make $P=C(\bar{u})$.

Step5: if the point $Q$ is not the original control point, we use the reverse node insertion method to make it a control point, denoted by $P_{k}$. In the direction of the line $P P_{k}$ taking a point $\tilde{P}$, calculating $d$.

Step6: according to formula (5) counting the $\tilde{\omega}_{k}$.

Step7: repeat steps 4 to 6 , getting the value of new weight factor corresponding to the change of the curve. Then the scope of convexity-preserving is obtained by observing the shape of the curve.

\subsection{Computation example}

A cubic NURBS curve interpolation is interpolated at the following data points $q_{0}=(-2.15,0.05)$ $q_{1}=(-0.45,0.45) \quad q_{2}=(0.14,1.5) \quad q_{3}=(0.13,1.81) \quad q_{4}=(0.85,2.30) \quad q_{5}=(1.50,2.39) \quad q_{6}=(1.83,1.85) \quad q_{7}=(0.90,1.66)$,the corresponding weight factors are $w_{0}=1, w_{1}=0.8, w_{2}=1, w_{3}=2.9, w_{4}=3.096, w_{5}=1.8, w_{6}=0.5$, $w_{7}=1.7, w_{8}=2.6, w_{9}=0.4$. Parameter value sequence is obtained after the date points are parameterized. $t_{k}=\left\{0, \frac{4}{25}, \frac{11}{25}, \frac{15}{25}, \frac{18}{25}, \frac{23}{25}, 1\right\}$ at the two endpoints adding the constraint of the endpoint vector $C^{\prime}(0)=(2.2,0.9), C^{\prime}(1)=(-6.1,-0.57)$.In order to rule out the influence of the direction of the endpoint vector and the improper length, we make the length of endpoint vector approximate the length of polygon of data points. By adjusting the direction and the length of the endpoint vector , we make them in the appropriate range get the node vectors $u=\left\{0,0,0,0, \frac{3}{8}, \frac{4}{8}, \frac{5}{8}, 1,1,1,1\right\}$ by the algorithm A-2.1, and calculate control point $P_{0}=(-0.445,0.118) \quad P_{1}=(0.013,0.128) \quad P_{2}=(1.140,1.200) \quad P_{4}=(1.831,2.147)$ $P_{3}=(0.011,1.420) P_{5}=(2.574,1.540) P_{6}=(3.050,1.282) P_{7}=(2.013,0.131) P_{8}=(1.970,0.760) P_{9}=(1.920,1.193)$ Cubic NURBS interpolation curves is obtained through the eight data points $q_{i}(i=0,1,2, \cdots, 7)$ based on the de Boor algorithm[12].

From the graph, we know that the convexity-preserving of curve corresponding to the control point $P_{4}$ is not ideal. We fixed all control points, except for the weight factor $w_{4}$, the other weight factors were kept constant, the shape of the curve was controlled by changing the value $w_{4}$. In the curve section corresponding to the control point $P_{4}$, we take a point $q$, so that the $q$ is moderately shifted to control the point $P_{4}$. The parameter values were calculated as $\bar{u}=0.52$. The curve is moved at the same increment $\delta=0.019$ to the control point $P_{4}$ at each time. Moving distance are $d=0.019, d=0.038, d=0.057, d=0.076$. Then, by using the formula (6), the value of the new weight factor corresponding to different moving distance can be calculated, and their values are $\tilde{\omega}_{4}^{1}=0.09, \tilde{\omega}_{4}^{2}=1.15, \tilde{\omega}_{4}^{3}=2.97$ and $\tilde{\omega}_{4}^{4}=3.20$ respectively. When the new power factor $\tilde{\omega}_{4}^{k}$ is greater than the weight factor $\tilde{\omega}_{4}^{4}$, the curve has a sharp point, which makes the NURBS interpolation curve is not convexity-preserving. 


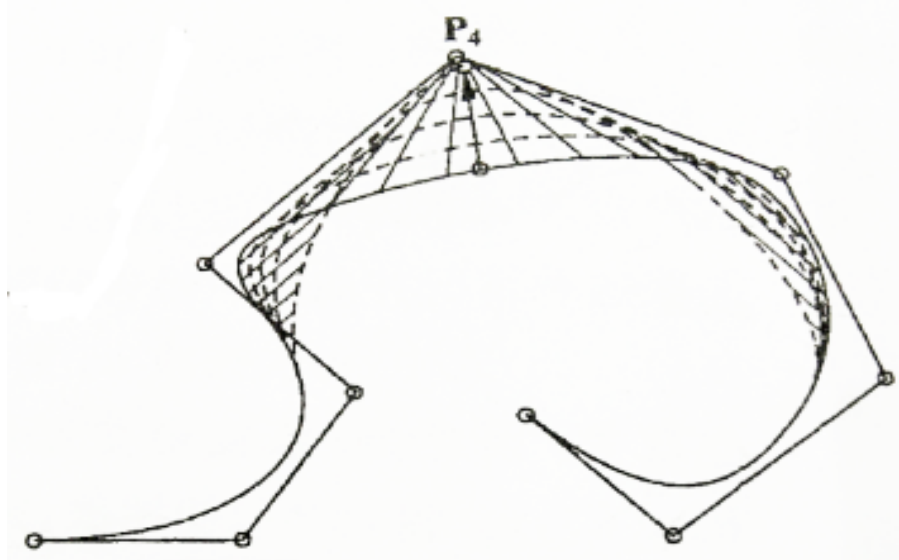

Figure3 the calculated new value of weight factor by modifying the weight factor are $\tilde{\omega}_{4}^{k}=\{0.09,1.15,2.97,3.096\}$ and the comparison- diagram of shape change of cubic

NURBS interpolation curves in $\bar{u}=0.52$

Form figure 3, the control of the shape of the 3 NURBS interpolation curve is modified based on the weight actor. Before the weights are changed, the curve is indicated by a solid line. The curve corresponding to modified new weights are showed by dotted lines. From the comparison chart when the weight actor is modified by a large margin, it leads to very bad parameterization. There is a cusp on the curve, so that the curve is not convexity-preserving. Hence, weight actors should be changed in a appropriate range. When the new values of weight actor are $\tilde{\omega}_{4}^{1}=0.09, \quad \tilde{\omega}_{4}^{2}=1.15, \tilde{\omega}_{4}^{3}=2.97$, the shape of the curve is ideal and has good convexity preserving. However, when the new weights value is $w_{4}=3.096$, the original curve have no cusp or loop, but the convexity-preserving of the curve is not very good. When the new weight value is $\tilde{\omega}_{4}^{4}=3.20$, curve is unsmooth or has cusp and it has not convexity-preserving, the curve appears deformity or loop with weights value increasing. Therefore, as the value of weight is limited in scope of $(0,3]$, the curve trend is ideal with good convexity-preserving. When the value of the weights is outside the range, Curve appear deformity and does not have convexity -preserving. So the scope of convexity-preserving of the NURBS interpolation curve is $(0,3]$ based on the weights.

\subsection{Application}

The design of the ship type line is very important in the design of the ship .How to improve the design level and design quality of the hull to reduce the cost of shipbuilding is always the pursuit of the shipyard. Due to the complex of Ship type line, every step of the design will be a long period. In design of ship type line, the importance of weights in the regulation of shape is self-evident. Adjusting the curve by changing the weights that is appropriate, can not only avoid a bad parameterization, but also shorten the design cycle and improve the design quality.

The following is the thirteenth station body lines of double stern ship line, with all control points and all weights except the $w_{i}$ fixed, only the weights $w_{i}$ is changed. Then observing the shape of the curve with the weights belongs to the scope of $(0,3]$ or not. Further the accuracy and practicality of convexity-preserving scope of the NURBS interpolation weights is verified.

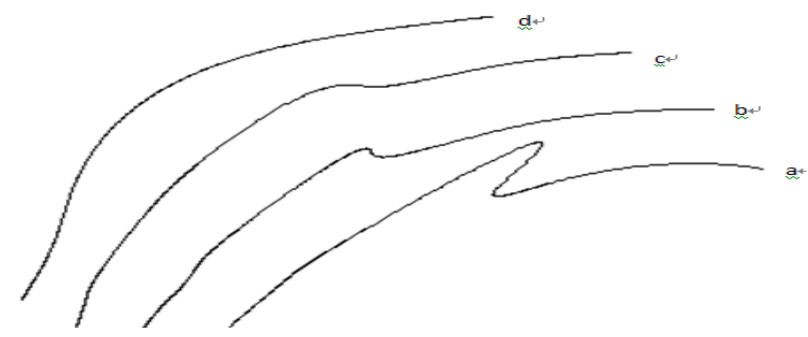

Figure 4 The comparison of cubic NURBS interpolation curve with weight factor given different values 
Figure 4 is the corresponding cubic NURBS interpolation curve with the weights to take different values. When $w_{j} \geq 10$, the shape of cubic NURBS interpolation curves is showed by $a$; When $w_{j}=6$, its shape is showed by $b$, When $w_{j}=3.5$, its shape is showed by $c$; When $w_{j}=3$, its shape is showed by $d$.That is, when the weights belong to the scope $(0,3]$, the approximate shape of the curve is similar to the curve $a, b, c$, when the weights not belong to the scope of $(0,3]$, the approximate shape of the curve is similar to the curve $d$, which curve trend is smooth and good convexity- preserving. The convexity-preserving scope of weights is $(0,3]$. When the appropriate change of the endpoint of the first order derivative and mode. The range of the convexity-preserving of weights is also $(0,3]$ under changing the constraint of the endpoint vector in a proper. Therefore, the scope of the convexity-preserving of the weight factor under the constraint of the endpoint vector is $(0,3]$. When designer use of NURBS method, the value of weights are changed in $(0,3]$, it not only can greatly reduce the workload and can be a good way to get the ideal curve model.

\section{Concluding remarks}

In this paper, the shape of NURBS curve is studied reversely. Based on the existing curve and surface interpolation, concrete steps of the convexity-preserving of the weight factor of cubic NURBS interpolation curve is given, through numerical example are carried out by computer simulation and the weights are controlled with purpose and procedure. A scope of the convexity-preserving is putted forward through observing the changes of the interpolation curve and the associated with this operation. Moreover, its application in the design of ship modeling is given. It is convenient for designers to control the weights rightly, and to make the weights of cautious application into free application, it is full of NURBS's potential. In addition, the working efficiency is improved and convenient to be used in practice.

\section{References:}

[1] Les A Piegl,Wayne Tiller :E\&C Vol.16-1 (2000),p.73-79.

[2] Imre Juhsz1 : CAGD Vol.16-5 (1999),p.377-383.

[3] PIEGL L : CAD Vol.21-8(1989),p.509-518.

[4] PIEGL L : IEEE CG\&A Vol.11-1(1991),p.55-71.

[5] AU C K, YUEN M F :CAD Vol.27-2(1995),p.85-93.

[6] Tiller, W:IEEE CG\&A Vol.19-9 (1983),p.485-498.

[7] L Piegl, W Tiller :The NURBS Book(Tsinghua University press,China2010).

[8] Les A Piegl, Khairan Rajab :E\& C Vol.24-1(2008),p.79-85.

[9] Chen S : MS\&T Vol.5-20(2001), p.692-693.

[10] Boehm, W: CAD Vol.12(1980), p.199-201.

[11] Snchez-Reyes J: IEEE CG\& AVol.17-1(1997),p.52-59.

[12] B K Choi: CAD Vol.22-4(1990),p.235-240. 